\title{
Curcumin Activates ROS Signaling to Promote Pyroptosis in Hepatocellular Carcinoma HepG2 Cells
}

\author{
WAN-FENG LIANG ${ }^{1 \#}$, YI-XI GONG ${ }^{1 \#}$, HAI-FENG LI $^{1}$, FU-LIANG SUN ${ }^{1}$, WEI-LONG LI ${ }^{2}$, DONG-QIN CHEN $^{2}$, \\ DAN-PING XIE ${ }^{2}, \mathrm{CHEN}_{-X I} \mathrm{REN}^{2}, \mathrm{XIAO-YU} \mathrm{GUO}{ }^{2}, \mathrm{ZI}^{-} \mathrm{YI} \mathrm{WANG}^{2}, \mathrm{TAEHO}_{\mathrm{KWON}}^{3}$ and HU-NAN SUN ${ }^{2}$ \\ ${ }^{1}$ Department of Veterinary Medicine, Agricultural College of Yanbian University, Yanji, P.R. China; \\ ${ }^{2}$ College of Life Science and Technology, Heilongjiang Bayi Agricultural University, Daqing, P.R. China; \\ ${ }^{3}$ Primate Resources Center, Korea Research Institute of Bioscience and Biotechnology (KRIBB), \\ Jeonbuk, Republic of Korea
}

\begin{abstract}
Background/Aim: Curcumin is a polyphenol that exerts a variety of pharmacological activities and plays an anti-cancer role in many cancer cells. It was recently reported that gasdermin E (GSDME) is involved in the progression of pyroptosis. Materials and Methods: HepG2 cells were treated with various concentrations of curcumin and cell viability was examined using MTT assay, apoptosis was analysed using flow cytometry, reactive oxygen species (ROS) levels using dihydroethidium, $L D H$ release using an $L D H$ cytotoxicity assay, and protein expression using western blot. Results: Curcumin increased the expression of the GSDME N-terminus and proteins involved in pyrolysis, promoted HspG2 cell pyrolysis and increased intracellular ROS levels. Moreover, inhibition of the production of intracellular ROS with nacetylcysteine (NAC) improved the degree of apoptosis and pyrolysis induced by curcumin. Conclusion: Curcumin induces HspG2 cell death by increasing apoptosis and pyroptosis, and ROS play a key role in this process. This study improves our understanding of the potential anti-cancer properties of curcumin in liver cancer.
\end{abstract}

This article is freely accessible online.

\#These Authors contributed equally to this work.

Correspondence to: Taeho Kwon, Ph.D., Primate Resources Center, Korea Research Institute of Bioscience and Biotechnology (KRIBB), 351-33 Neongme-gil, Ibam-myeon, Jeongeup-si, Jeonbuk, 56216, Republic of Korea. Tel: +82 635705316, Fax: +82 635705309, e-mail: kwon@kribb.re.kr; Hu-Nan Sun, Ph.D., College of Life Science \& Technology, Heilongjiang Bayi Agricultural University, Xinyanglu \#2, 163319, Daqing, P.R. China. Tel: +86 4596819300, Fax: +86 4596819295, e-mail: sunhunan76@163.com

Key Words: Curcumin, GSDME, liver cancer, proptosis, ROS.
Curcumin [1,7-bis-(4-hydroxy-3-methoxyphenyl)-1,6heptadiene-3,5-dione (diferuloy 1 methane)] is a type of polyphenol extracted from the rhizomes of ginger plants, such as turmeric and zedoary (1), which has been shown to display various pharmacological activities, including antiinflammatory (2), antioxidant (3), hypolipidemic (4), antitumor (5), and immune regulatory (6). Previous studies have also demonstrated that curcumin exerts anti-cancer effects in many types of cancer cells, including stomach (7), colon (8), lung (9), liver (10) and breast (11). In addition, it has been reported that curcumin can affect the cellular redox balance, resulting in increased cellular reactive oxygen species (ROS) production (12). Excessive ROS can directly or indirectly affect the regulation of anti- or pro-apoptotic proteins by Bcl-2 family members, leading to cellular apoptosis (13). Moreover, ROS accumulation has been shown to induce cellular apoptosis, necroptosis, endoplasmic reticulum stress, and autophagy in various cancer cells (14). Therefore, curcumin may also affect cell death in liver cancer cells.

Pyroptosis is a recently discovered type of programmed cell death that depends on the activation of the inflammationrelated caspases $1,4,5$, and 11 , as well as apoptosis-related caspase-3 (15). The morphology of cells undergoing pyroptosis differs from that in apoptosis or necrosis, and is characterized by cell swelling, membrane breakage, pore formation, and osmotic lysis (16). It was recently reported that gasdermin E (GSDME) is involved in the progression of pyroptosis (17). In particular, activated caspase-3 was found to cleave GSDME between the N-terminus (GSMDE-N) and C-terminus under certain apoptotic stimuli, thus relieving the self-inhibition of the C-terminus and releasing the active GSDME-N-terminus as well as the pore-forming domain (PFD). Consequently, nonselective pores are formed in the cell membrane, leading to cell swelling, membrane rupture, and cell pyrolysis (18). However, the effect of curcumin on GSDME during pyroptotic cell death in liver cancer cells remains unclear. 
In this study, the effect of curcumin on cell death was evaluated by examining the viability, apoptosis, pyroptosis, and expression of related proteins in HepG2 liver cancer cells treated with curcumin. Together, the findings of this study provide new insights into the potential anti-cancer properties of curcumin in liver cancer.

\section{Materials and Methods}

Chemicals and materials. Dulbecco's modified Eagle's medium (DMEM), fetal bovine serum (FBS), and Annexin V apoptosis detection kit FITC were purchased from Invitrogen (Carlsbad, CA, USA). Penicillin-streptomycin liquid $(\mathrm{P} / \mathrm{S}), 1 \times$ tris-buffered saline and Tween 20 (TSBT) buffer solution, n-acetylcysteine (NAC), and Hoechst 32258 were obtained from Solarbio Life Sciences (Beijing, China). Propidium iodide (PI) staining kit, horseradish peroxidase (HP)conjugated goat anti-rabbit IgG and anti-mouse IgG were purchased from Sangon Biotech (Shanghai, China). 3-(4,5-dimethylthiazol-2-yl)2,5-diphenyltetrazolium bromide (MTT) and curcumin were obtained from Sigma-Aldrich (St. Louis, MO, USA). Six- and ninety-six-well plates were purchased from NEST Biotechnology (Wuxi, Jiangsu, PR China). Dihydroethidium (DHE) and LDH cytotoxicity assay kits were obtained from Beyotime Biotechnology (Shanghai, PR China). AntiBax (ab32503), anti-Bcl-2 (ab32124), anti-caspase-3 (ab32351), antiDFN45/GSDME (ab215191), and anti- $\alpha$ tubulin (ab7291) antibodies were purchased from Abcam (Cambridge, MA, USA). The chemiluminescence detection system was obtained from GE Healthcare Life Sciences (Chalfont, UK). The UV MAX kinetic microplate reader was purchased from Molecular Devices (LLC, Sunnyvale, CA, USA). Fluorescence microscopy was performed using an $\operatorname{EVOS}^{\circledR} \mathrm{xl}$ core cell culture microscope from the Advanced Microscopy Group (Paisley, Scotland). Flow cytometry was performed using a FACSCalibur instrument from BD Biosciences (Franklin Lakes, NJ, USA). Nitrocellulose membranes were obtained from Millipore (Bedford, MA, USA).

Cell culture. HepG2 liver cancer cells were cultured in DMEM supplemented with $10 \% \mathrm{FBS}$ and $1 \% \mathrm{P} / \mathrm{S}$, and maintained in a humidified incubator at $37^{\circ} \mathrm{C}$ with $5 \% \mathrm{CO}_{2}$.

Cell viability assay. Cell viability was analyzed using an MTT assay. Briefly, HepG2 cells were seeded in 96-well plates $\left(1 \times 10^{4}\right.$ cells/well) and treated with curcumin for $12 \mathrm{~h}$. Next, $10 \mu \mathrm{l}(0.5$ $\mathrm{mg} / \mathrm{ml}$ ) of MTT was added to each well and incubated for $2 \mathrm{~h}$ at $37^{\circ} \mathrm{C}$ in $5 \% \mathrm{CO}_{2}$. After the supernatant had been removed, $100 \mu \mathrm{l}$ of dimethyl sulfoxide was added and absorbance was measured at $490 \mathrm{~nm}$ using a UV MAX kinetic microplate reader.

Apoptosis detection. HepG2 cells were seeded in 6-well plates $(2 \times$ 105 cells/well) and treated with curcumin. To detect apoptosis, cells were prepared using an Annexin V apoptosis detection kit FITC according to the manufacturer's protocol and analyzed using fluorescence microscopy and flow cytometry.

ROS detection. HepG2 cells were seeded in 6-well plates $\left(2 \times 10^{5}\right.$ cells/well) and treated with curcumin. Changes in cellular ROS levels were determined using DHE $(1 \mu \mathrm{M} / \mathrm{ml})$ and nuclei were visualized qualitatively under a microscope after $20 \mathrm{~min}$ of incubation with Hoechst 32258 dye $(2 \mu \mathrm{g} / \mathrm{ml})$.
Detection of LDH release. HepG2 cells were seeded in 96-well plates $\left(1 \times 10^{5}\right.$ cells/well $)$ and treated with curcumin. Changes in LDH release were determined using an LDH cytotoxicity assay. Absorbance was measured at $490 \mathrm{~nm}$ using a UV MAX kinetic microplate reader, according to the manufacturer's instructions.

Lytic cell death assay. HepG2 cells were seeded in 6-well plates $\left(2 \times 10^{5}\right.$ cells/well $)$ and treated with curcumin. Lytic cell death was measured using PI incorporation, as described previously. Nuclei were visualized using a fluorescence microscope after incubation with Hoechst 33342 dye $(2 \mu \mathrm{g} / \mathrm{ml})$ and PI for $20 \mathrm{~min}$ at room temperature (19).

Western blot analysis. Cell lysate proteins were separated using 12 $\%$ sodium dodecyl sulfate-polyacrylamide gels and transferred onto nitrocellulose membranes, which were blotted with primary antibodies against Bax, Bcl-2, caspase-3, GSDME, and $\alpha$-tubulin at $4^{\circ} \mathrm{C}$ for $12 \mathrm{~h}$. The membranes were then washed five times with TBST and incubated with HP-conjugated goat anti-rabbit or antimouse $\mathrm{IgG}$ for $2 \mathrm{~h}$ at room temperature. After excess antibodies were removed by washing with TBST, specific binding was detected using a chemiluminescence detection system according to the manufacturer's protocol.

Statistical analysis. Data from at least three independent experiments are presented as the mean \pm standard error of the mean (SEM). Two-way analysis of variance (ANOVA) was used to analyze changes over time and differences between groups in each experiment. For most experiments, Tukey's post hoc test $(\alpha=0.05)$ was used to determine statistical significance among two groups. All statistical analyses were conducted using SPSS software (version 25; IBM Corp). Differences were considered statistically significant if the p-value was less than $0.05(* p<0.05, * * p<0.01$, $* * * p<0.001)$.

\section{Results}

Curcumin increased cellular ROS levels and induced apoptosis in HepG2 cells. The chemical structure of curcumin is shown in Figure 1A. To determine its effect on HepG2 cell viability, cells were treated with various concentrations of curcumin $(0,5,10,20$, or $30 \mu \mathrm{M})$ for 12 $\mathrm{h}$ and cell viability was examined using an MTT assay. Curcumin significantly reduced the viability of HepG2 cells in a dose-dependent manner (Figure 1B). Furthermore, the effect of curcumin on apoptosis induction was investigated by treating HepG2 cells with different curcumin concentrations $(0,20$, or $30 \mu \mathrm{M})$ for $12 \mathrm{~h}$ and with $20 \mu \mathrm{M}$ of curcumin for different durations $(0,3,6,9,12$, or $24 \mathrm{~h})$. Cellular apoptosis was evaluated using an Annexin-V FITC detection kit and flow cytometry, and revealed that curcumin significantly increased apoptosis in HepG2 cells in a dose- and time-dependent manner (Figure 1C and D). The effect of curcumin on the cellular ROS levels was also examined using fluorescence microscopy and flow cytometry by staining with DHE (a superoxide detection marker) after HepG2 cells had been treated with different 
A<smiles>COc1cc(/C=C/C(=O)CC(=O)/C=C/c2ccc(O)c(OC)c2)ccc1O</smiles>

\section{Curcumin}

C

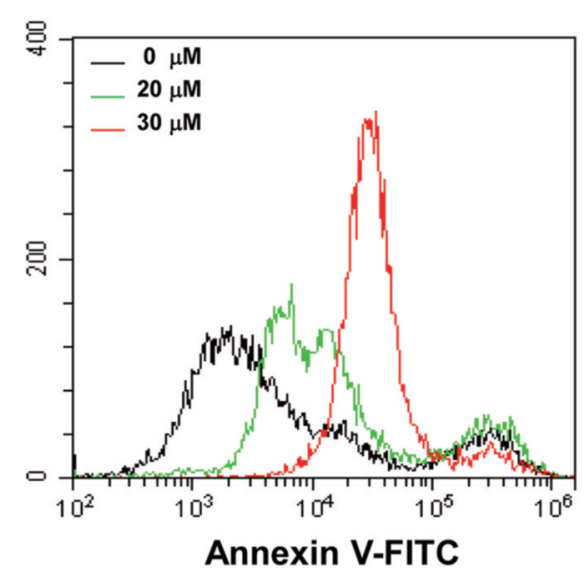

E

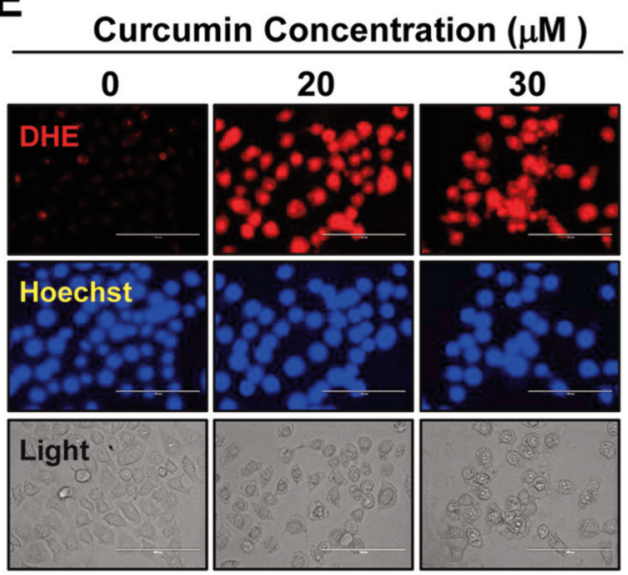

B

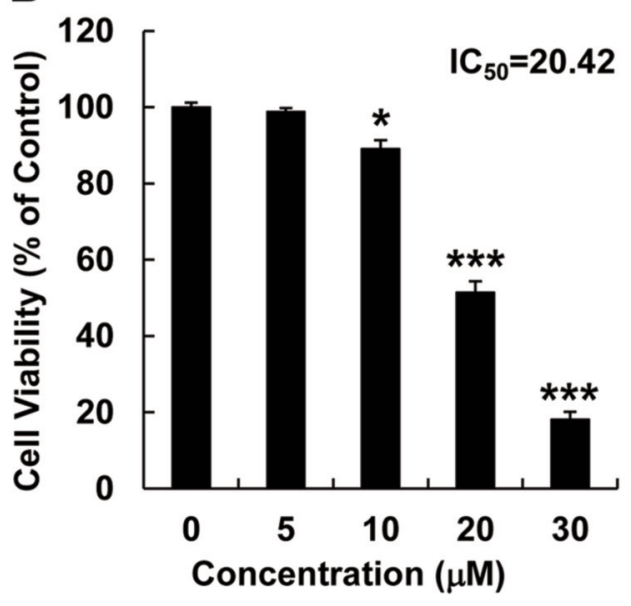

D

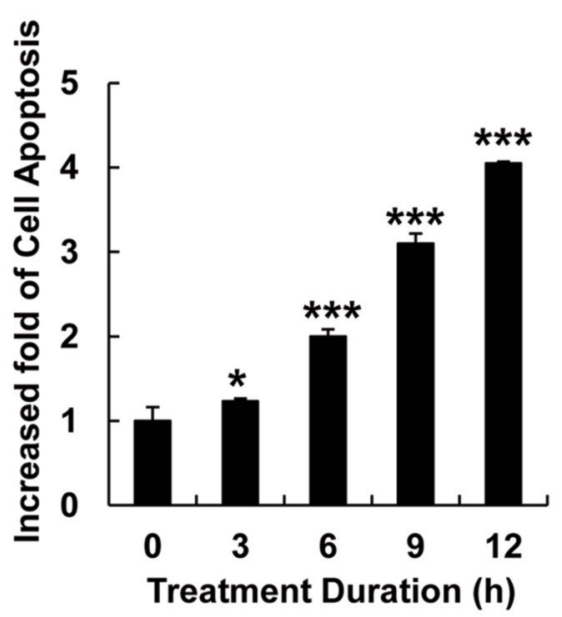

F

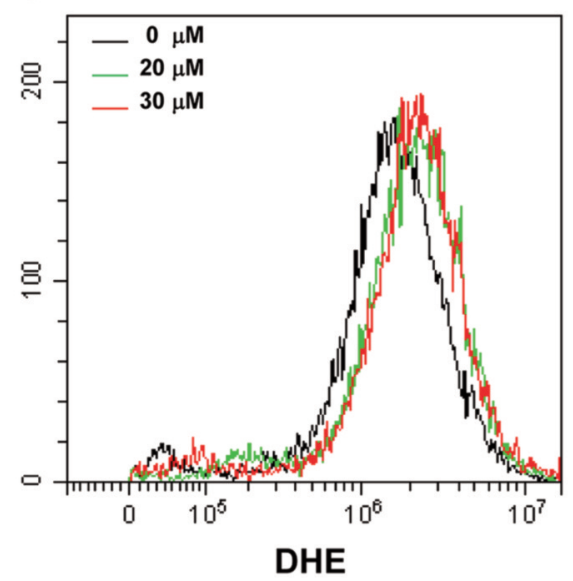

Figure 1. Curcumin increased cellular ROS levels and induced apoptosis in HepG2 liver cancer cells. (A) The chemical structural of curcumin. (B) Cell viability was analyzed using MTT assay, in HepG2 cells treated with curcumin (0, 5, 10, 20, or $30 \mu M)$ for 12 h. (C) Cellular apoptosis was measured using flow cytometry in HepG2 cells treated with curcumin $(0,20$, or $30 \mu \mathrm{M})$ for $12 \mathrm{~h}$. Black line: without curcumin; green line: $20 \mu \mathrm{M}$ curcumin; red line: $30 \mu M$ curcumin. (D) Cellular apoptosis was measured by flow cytometry in HepG2 cells treated with curcumin at different times $(0,3,6$, or $12 \mathrm{~h})$. The fold increase in apoptotic cells is presented as the mean $\pm \operatorname{SEM}\left({ }^{*} p<0.05,{ }^{*} p<0.01\right)$. (E) Cellular ROS levels were detected by fluorescence microscopy in DHE (red)- and Hoechst (blue)-stained HepG2 cells treated with curcumin (0, 20, or $30 \mu \mathrm{M})$ for $12 \mathrm{~h}$ (scale bar=100 $\mu \mathrm{m})$. (F) Cellular ROS levels were detected by fluorescence microscopy in DHE-stained HepG2 cells treated with curcumin (0, 20, or 30 $\mu$ M) for 12 h. 
A
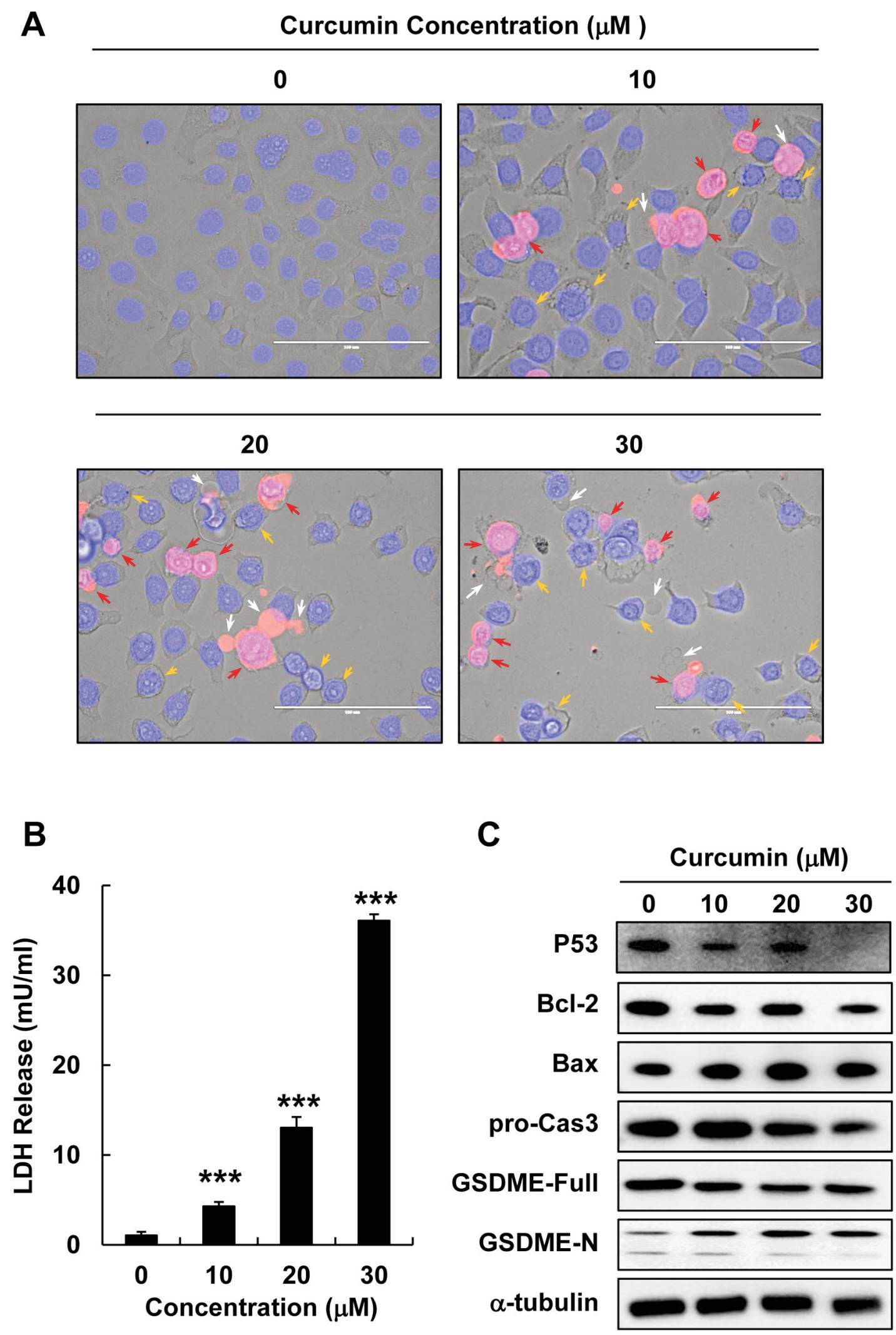

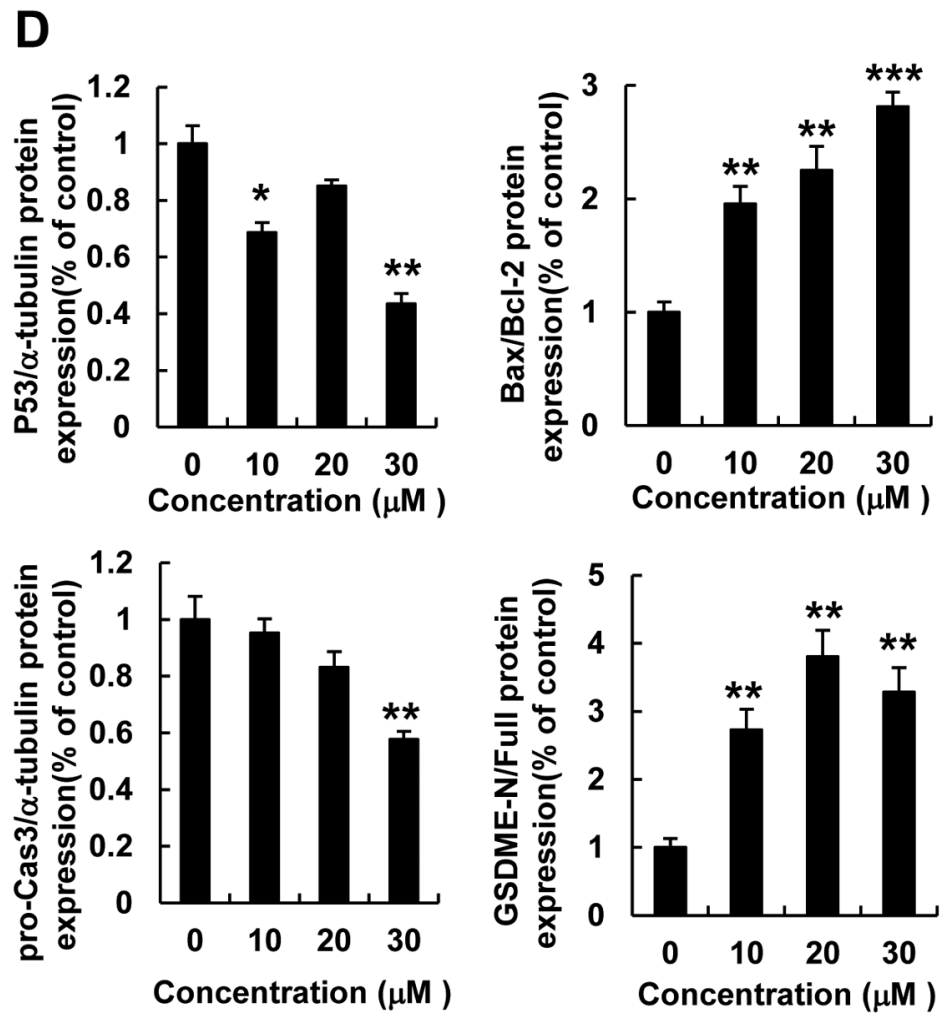

Figure 2. Curcumin induced lytic cell death and pyroptosis in HepG2 cells. HepG2 cells were treated with curcumin (0, 10, 20, or 30 $\mu$ M) for 12 h. (A) Morphological changes and pyroptosis were observed using fluorescence microscopy in PI (red)- and Hoechst (blue)-stained HepG2 cells treated with curcumin $(0,10,20$, or $30 \mu \mathrm{M})($ scale bar $=100 \mu \mathrm{m})$. (B) LDH release in HepG2 cells treated with curcumin $(0,10,20$, or $30 \mu \mathrm{M})$. (C) Bcl-2, Bax, pro-caspase3, GSDME-Full, and GSDME-N protein expression detected using western blot analysis in HepG2 cells treated with curcumin $(0,10,20$, or $30 \mu M)$. (D) Quantitative analysis of Bcl-2, pro-caspase3, and GSDME-N protein expression presented as the mean $\pm S E M$ of three different samples $(* p<0.05, * * p<0.01, * * * p<0.001)$.

curcumin concentrations $(0,20$, or $30 \mu \mathrm{M})$. Curcumin significantly increased intracellular ROS levels in HepG2 cells (Figure 1E and F).

Curcumin induced lytic cell death and pyroptosis in HepG2 cells. To elucidate the features of cell death induced by curcumin, we performed PI and Hoechst staining in HepG2 cells treated with different curcumin concentrations $(0,10$, 20 , or $30 \mu \mathrm{M}$ ) for $12 \mathrm{~h}$. The observation of morphological changes using a fluorescent microscope revealed swelling, membranolysis, and the formation of numerous pyroptotic bodies in curcumin-treated HepG2 cells (Figure 2A). LDH release is a marker of cell death that is closely related to cell membrane destruction; therefore, we examined LDH release in HepG2 cells treated with various curcumin concentrations $(0,10,20$, or $30 \mu \mathrm{M})$ for $12 \mathrm{~h}$. Curcumin significantly increased LDH release in HepG2 cells in a dose-dependent manner (Figure 2B), indicating that curcumin treatment increases cell death. In addition, western blotting analysis revealed that curcumin treatment significantly down- regulated pro-caspase 3 and $\mathrm{Bcl}-2$ (anti-apoptotic) protein expression but up-regulated $\mathrm{Bax}$ (pro-apoptotic) protein expression in HepG2 cells (Figure 2C and D). Next, we measured the expression of the GSDME protein, which is a known marker of pyroptosis, after curcumin treatment, and found that increasing curcumin concentrations downregulated full length GSDME and up-regulated GSDME-N (Figure 2C and D). Thus, curcumin treatment appears to increase pyroptosis in HepG2 cells.

ROS inhibition reduced cellular apoptosis and LDH release. To verify the regulatory effect of curcumin-induced ROS levels on cell death, HspG2 cells were pre-treated with NAC (a ROS scavenger) for $30 \mathrm{~min}$ and then, treated with curcumin for $12 \mathrm{~h}$. Flow cytometry and fluorescence microscopy analyses revealed that NAC treatment significantly reduced curcumin-stimulated cellular ROS levels, as detected using DHE staining (Figure 3A and B). In addition, NAC treatment reversed the reduction in cell viability induced by curcumin in HepG2 cells (Figure 3C); 
A

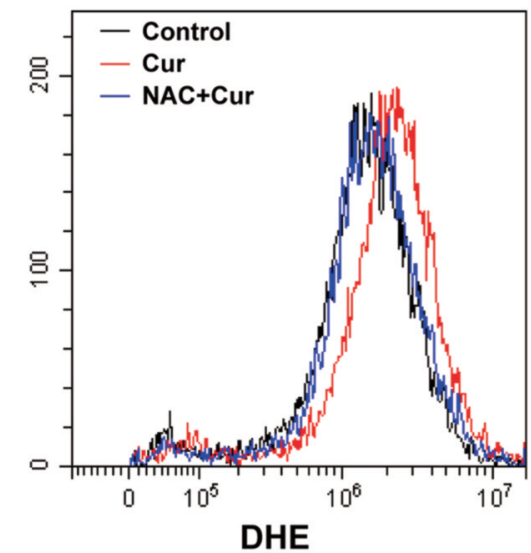

C

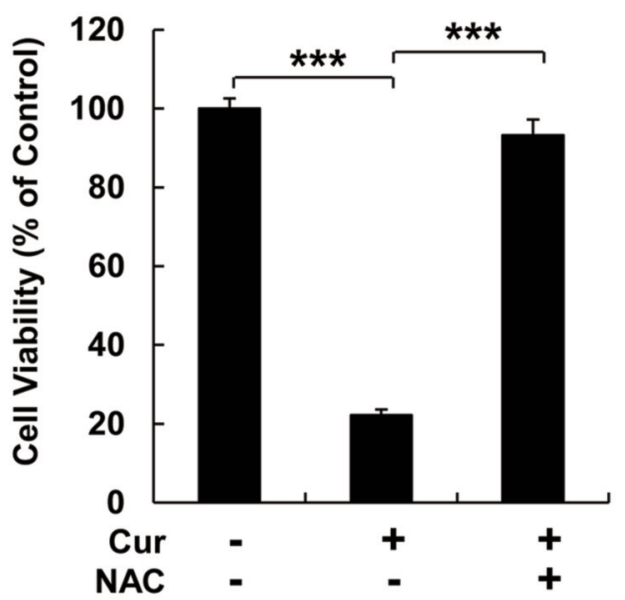

E

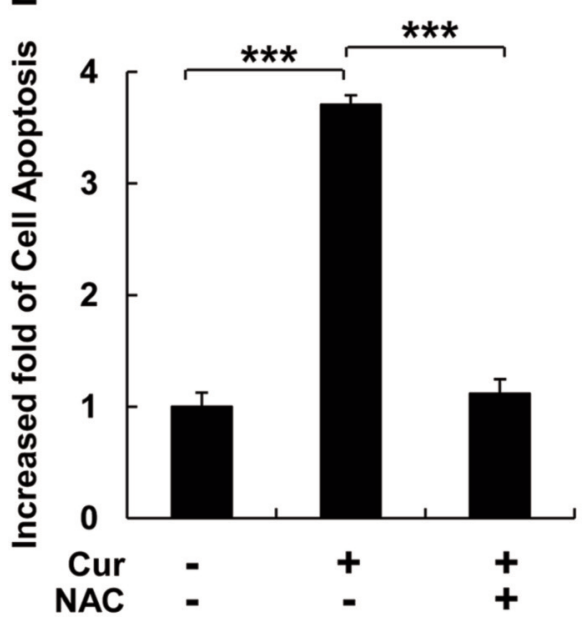

B

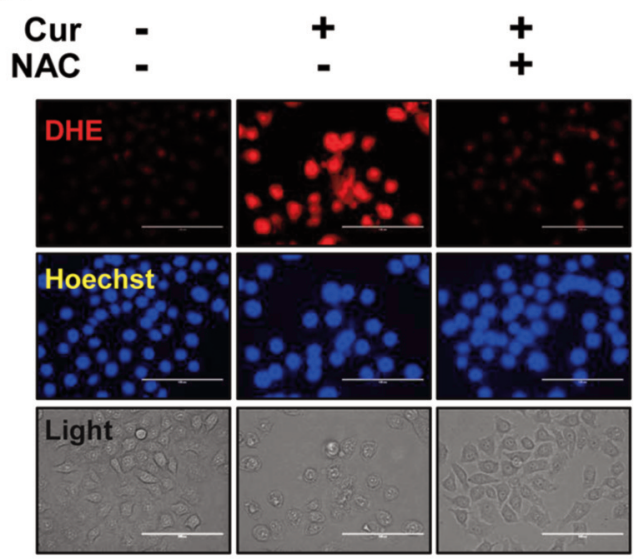

D

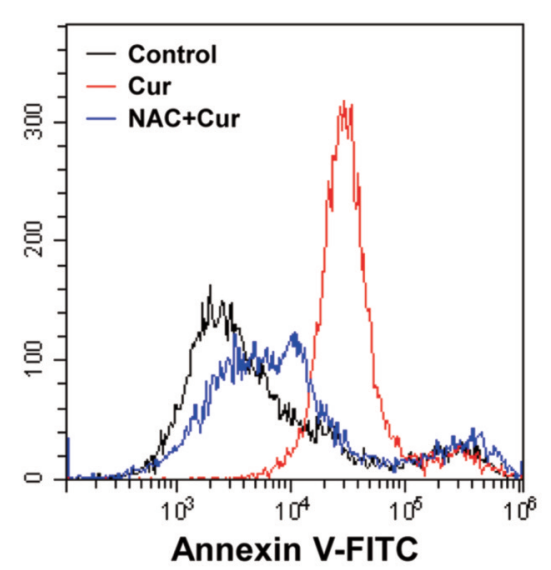

F

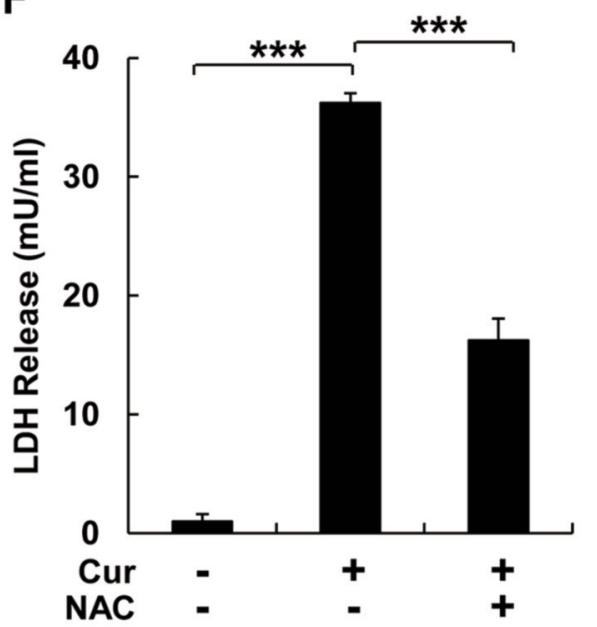

Figure 3. ROS inhibition reduces cellular apoptosis and LDH release. HepG2 cells were pre-treated with NAC, a ROS scavenger, for 30 min. (A) Cellular ROS levels were detected using flow cytometry with DHE staining. Black: no treatment; red: curcumin treatment; blue: NAC pretreatment and curcumin treatment. (B) Cellular ROS were detected using a fluorescence micrograph with DHE (red) and Hoechst (blue) staining (scale bar=100 $\mu \mathrm{m})$. (C) Cell viability was analyzed using an MTT assay. (D) Cellular apoptosis was detected using flow cytometry and Annexin V - FITC staining of HepG2 cells. Black: normal cells; red: curcumin treatment; blue: NAC pretreatment and curcumin treatment. (E) Quantitative analysis of cellular apoptosis shown in $(D) .(F)$ LDH production in HepG2 cells following three different treatments, as indicated. Data represent the mean \pm SEM of three different samples (**** $<0.001)$. 
A

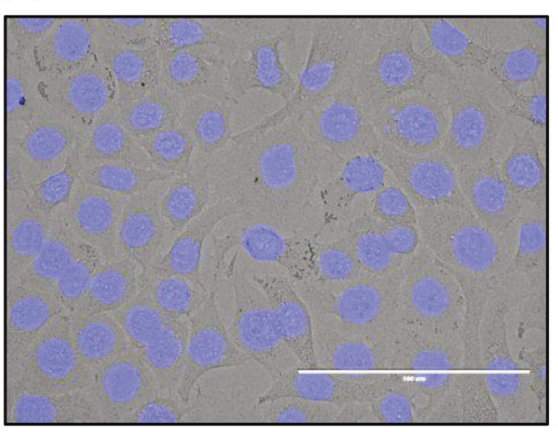

B

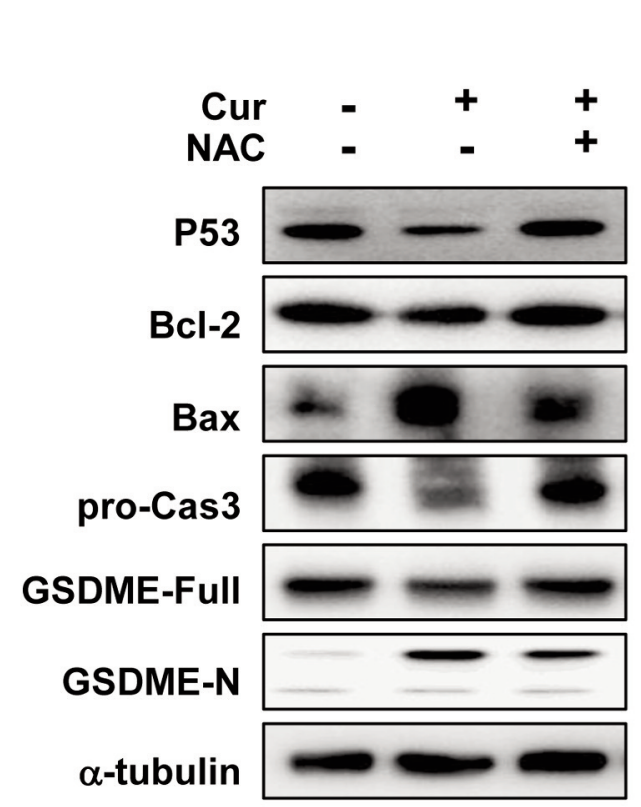

Cur

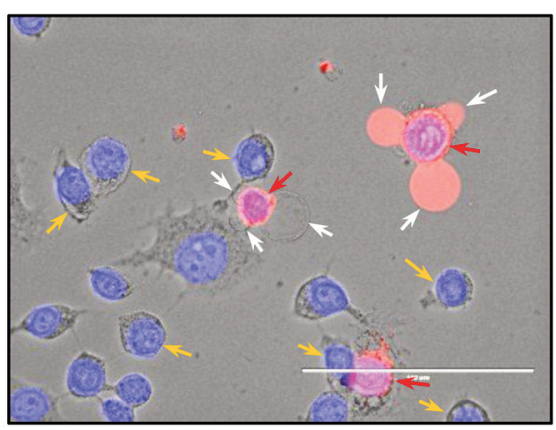

C
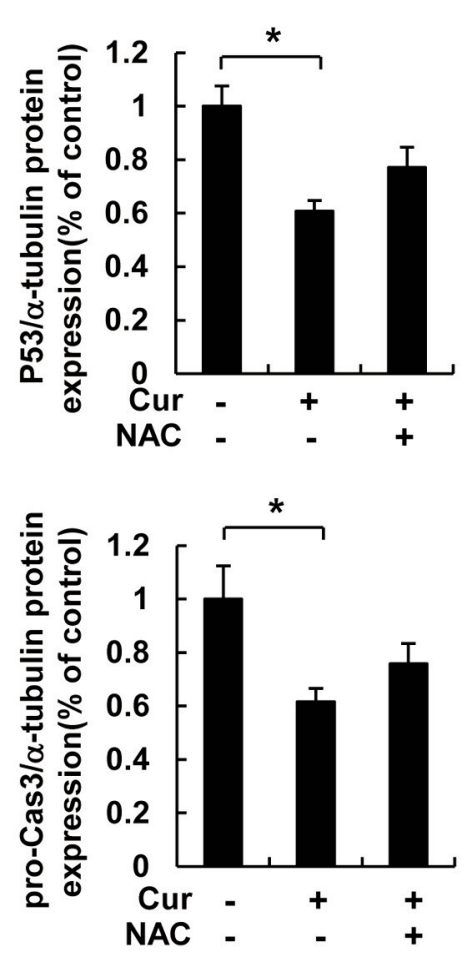

NAC+Cur

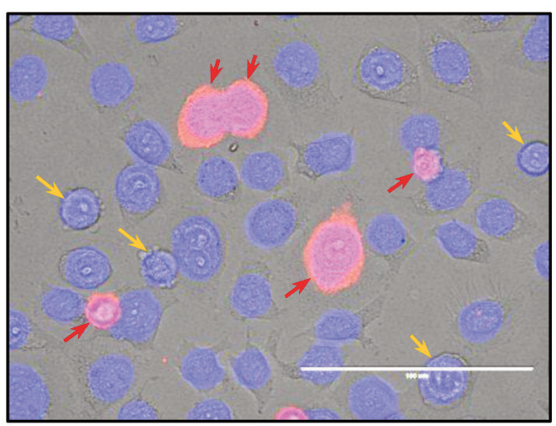

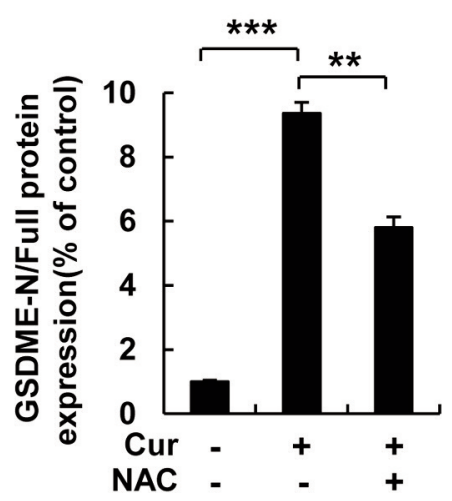

Figure 4. ROS inhibition reduces lytic cell death and pyroptosis. HepG2 cells were pretreated with NAC for 30 min and then, treated with curcumin for 12 h. (A) Morphological changes and pyroptosis were observed using fluorescence microscopy of PI (red)- and Hoechst (blue)-stained HepG2 cells treated with curcumin $(30 \mu M)$ (scale bar=100 $\mu \mathrm{m})$. (B) Bcl-2, Bax, pro-caspase3, GSDME-Full, and GSDME-N protein expression was detected using western blot analysis in HepG2 cells treated with curcumin. (C) Quantitative analysis of Bcl-2, pro-caspase3, and GSDME-N protein expression presented as the mean \pm SEM of three different samples $\left({ }^{*} p<0.05\right.$, **p $<0.01$, ***p<0.001).

therefore, we examined the effect of NAC on curcumininduced cellular apoptosis and pyroptosis in HepG2 cells. NAC treatment significantly reduced curcumin-induced cell apoptosis and LDH release in HepG2 cells (Figure 3D-F).

ROS play a key role in curcumin-induced HepG2 cell pyroptosis. To verify the regulatory role of ROS in curcumin-induced pyroptosis, HepG2 cells were pre-treated with NAC for $30 \mathrm{~min}$, treated with curcumin for $12 \mathrm{~h}$, and morphological changes were observed using fluorescence microscopy after staining with PI and Hoechst (Figure 4A). NAC treatment reversed curcumin-induced cell swelling, membranolysis, and pyroptotic body formation in HepG2 cells; therefore, we examined protein expression in HepG2 cells following curcumin and NAC treatment to identify the molecular mechanism of ROS in curcumin-induced pyroptosis. Interestingly, NAC treatment reversed the downregulation of pro-caspase 3 and $\mathrm{Bcl}-2$ and up-regulation of 
Bax protein induced by curcumin in HepG2 cells, as well as the down-regulation of full length GSDME and up-regulation of GSDME-N (Figure 4B and C).

\section{Discussion}

Liver cancer is one of the most common tumors worldwide, with around one million individuals being diagnosed each year (20). Current treatments for liver cancer mainly involve surgical resection and chemotherapy (21); however, both of these methods have limitations. For instance, surgical resection can only be conducted in a small proportion of patients, and chemotherapy can also kill normal cells in addition to cancer cells, causing adverse side effects. Therefore, it is necessary to find novel, safe and effective treatment strategies for liver cancer.

The use of herbal medicines has been recorded in pharmacological papers dating back to ancient Korea and China (22); however, traditional Chinese herbal medicine has attracted interest from scientists due to their high quantity and variety, good safety, and low price (23). In-depth research has revealed that Korean and Chinese herbal medicines and their extracts can exert good inhibitory effects against cancer at all stages of development (24). For instance, quercetin is a natural flavonoid that can inhibit proliferation and metastasis of a variety of tumor cells (25), while salidroside has been shown to inhibit the proliferation of A549 lung cancer cells (26). Similarly, Salvia miltiorrhiza can inhibit the over-expression of the proto-oncogene c-myc (27), and curcumin has been found to inhibit cancer cell proliferation by inhibiting DNA synthesis (28). Thus, Chinese herbal medicine extracts can exert a variety of anti-cancer effects.

Curcumin is a type of polyphenol extracted from the rhizome of ginger plants that has been shown to exert anticancer effects, such as inhibition of cell proliferation and induction of apoptosis via ROS (29). Consistent with previous research, our study demonstrated that curcumin can effectively induce apoptosis in HepG2 liver cancer cells in a time- and concentration-dependent manner. Moreover, morphological observation revealed that curcumin treatment causes cells to swell, form pores, and release $\mathrm{LDH}$, suggesting that curcumin induces pyrolysis in HepG2 cells.

Pyroptosis is a recently discovered form of programmed cell death characterized by swelling that results in cell membrane rupture, the release of cell contents, and the activation of a strong inflammatory response. Previous studies have indicated that small-molecule drugs can induce pyrolysis by mediating various cell signaling pathways involving proteins of the GSDM family (30). In this study, we found that curcumin can increase GSDME-N expression as well as the expression of proteins involved in pyrolysis, thereby promoting pyrolysis. In addition, we revealed that curcumin can increase intracellular ROS levels, suggesting that inhibition $\mathrm{f}$ intracellular ROS production can promote the degree of apoptosis and pyrolysis induced by curcumin. Taken together, our results demonstrate that curcumin can promote apoptosis and pyroptosis in liver cancer cells by regulating ROS production, thus exerting anticancer effects.

In conclusion, our results suggest that curcumin can induce liver cancer cell death by increasing apoptosis and pyroptosis, and that ROS play a key role in this process. In addition, curcumin activates the GSDME-related scorch death and mitochondria-dependent apoptosis signaling pathways. Thus, curcumin may be a potential new strategy for treating liver cancer.

\section{Conflicts of Interest}

The Authors declare that there are no conflicts of interest regarding this study.

\section{Authors' Contributions}

Conceptualization: WFL, YXG, TK, HNS. Methodology: TK, HNS Software: TK, HNS. Validation: TK, HNS. Formal Analysis: HFL, FLS, WLL, DQC, DPX, CXR. Investigation: WFL, YXG, TK, HNS. Resources: TK, HNS. Data Curation: TK, HNS. Writing Original Draft: WFL, YXG, TK, HNS. Writing - Review \& Editing: TK, HNS. Visualization: TK, HNS. Supervision: TK, HNS. Project Administration: TK, HNS. Funding Acquisition: TK. All Authors read and approved the final article.

\section{Acknowledgements}

This research was supported by Basic Science Research Program through the National Research Foundation of Korea (NRF) funded by the Ministry of Education (2020R1I1A2052417) and by grants from the Korean Research Institute of Bioscience and Biotechnology Research Initiative Program (KRIBB) (RBM0112011), Republic of Korea.

\section{References}

1 Nelson KM, Dahlin JL, Bisson J, Graham J, Pauli GF and Walters MA: The essential medicinal chemistry of curcumin. J Med Chem 60(5): 1620-1637, 2017. PMID: 28074653. DOI: 10.1021/acs.jmedchem.6b00975

2 Menon VP and Sudheer AR: Antioxidant and anti-inflammatory properties of curcumin. Adv Exp Med Biol 595: 105-125, 2007. PMID: 17569207. DOI: 10.1007/978-0-387-46401-5_3

3 Abrahams S, Haylett WL, Johnson G, Carr JA and Bardien S: Antioxidant effects of curcumin in models of neurodegeneration, aging, oxidative and nitrosative stress: A review. Neuroscience 406: 1-21, 2019. PMID: 30825584. DOI: 10.1016/j.neuro science.2019.02.020

4 Zingg JM, Hasan ST and Meydani M: Molecular mechanisms of hypolipidemic effects of curcumin. Biofactors 39(1): 101-121, 2013. PMID: 23339042. DOI: 10.1002/biof.1072

5 Unlu A, Nayir E, Dogukan Kalenderoglu M, Kirca O and Ozdogan M: Curcumin (turmeric) and cancer. J buon 21(5): 1050-1060, 2016. PMID: 27837604. 
6 Mollazadeh H, Cicero AFG, Blesso CN, Pirro M, Majeed M and Sahebkar A: Immune modulation by curcumin: The role of interleukin-10. Crit Rev Food Sci Nutr 59(1): 89-101, 2019. PMID: 28799796. DOI: 10.1080/10408398.2017.1358139

7 Sun C, Zhang S, Liu C and Liu X: Curcumin promoted mir-34a expression and suppressed proliferation of gastric cancer cells. Cancer Biother Radiopharm 34(10): 634-641, 2019. PMID: 31539270. DOI: $10.1089 / \mathrm{cbr} .2019 .2874$

8 Zhu J, Zhao B, Xiong P, Wang C, Zhang J, Tian X and Huang Y: Curcumin induces autophagy via inhibition of yes-associated protein (yap) in human colon cancer cells. Med Sci Monit 24: 7035-7042, 2018. PMID: 30281585. DOI: 10.12659/MSM.910650

9 Wan Mohd Tajuddin WNB, Lajis NH, Abas F, Othman I and Naidu R: Mechanistic understanding of curcumin's therapeutic effects in lung cancer. Nutrients 11(12): 2989, 2019. PMID: 31817718. DOI: 10.3390/nu11122989

10 Ren B, Luo S, Tian X, Jiang Z, Zou G, Xu F, Yin T, Huang Y and Liu J: Curcumin inhibits liver cancer by inhibiting damp molecule hsp70 and tlr4 signaling. Oncol Rep 40(2): 895-901, 2018. PMID: 29901164. DOI: 10.3892/or.2018.6485 Abstract

11 Calaf GM, Ponce-Cusi R and Carrión F: Curcumin and paclitaxel induce cell death in breast cancer cell lines. Oncol Rep 40(4): 2381-2388, 2018. PMID: 30066930. DOI: 10.3892/or.2018.6603

12 Larasati YA, Yoneda-Kato N, Nakamae I, Yokoyama T, Meiyanto E and Kato JY: Curcumin targets multiple enzymes involved in the ros metabolic pathway to suppress tumor cell growth. Sci Rep 8(1): 2039, 2018. PMID: 29391517. DOI: 10.1038/s41598-018-20179-6

13 Chong SJ, Low IC and Pervaiz S: Mitochondrial ros and involvement of bcl-2 as a mitochondrial ros regulator. Mitochondrion 19(Pt A): 39-48, 2014. PMID: 24954615. DOI: 10.1016/j.mito.2014.06.002

14 Ryter SW, Kim HP, Hoetzel A, Park JW, Nakahira K, Wang X and Choi AM: Mechanisms of cell death in oxidative stress. Antioxid Redox Signal 9(1): 49-89, 2007. PMID: 17115887. DOI: 10.1089/ars.2007.9.49

15 Shi J, Gao W and Shao F: Pyroptosis: Gasdermin-mediated programmed necrotic cell death. Trends Biochem Sci 42(4): 245254, 2017. PMID: 27932073. DOI: 10.1016/j.tibs.2016.10.004

16 Frank D and Vince JE: Pyroptosis versus necroptosis: Similarities, differences, and crosstalk. Cell Death Differ 26(1): 99-114, 2019. PMID: 30341423. DOI: 10.1038/s41418-018-0212-6

17 Wang Y, Gao W, Shi X, Ding J, Liu W, He H, Wang K and Shao F: Chemotherapy drugs induce pyroptosis through caspase-3 cleavage of a gasdermin. Nature 547(7661): 99-103, 2017. PMID: 28459430. DOI: 10.1038/nature22393

18 Kovacs SB and Miao EA: Gasdermins: Effectors of pyroptosis. Trends Cell Biol 27(9): 673-684, 2017. PMID: 28619472. DOI: 10.1016/j.tcb.2017.05.005

19 Zhang CC, Li CG, Wang YF, Xu LH, He XH, Zeng QZ, Zeng CY, Mai FY, Hu B and Ouyang DY: Chemotherapeutic paclitaxel and cisplatin differentially induce pyroptosis in a549 lung cancer cells via caspase-3/gsdme activation. Apoptosis 24(3-4): 312-325, 2019. PMID: 30710195. DOI: 10.1007/s 10495-019-01515-1
20 Sia D, Villanueva A, Friedman SL and Llovet JM: Liver cancer cell of origin, molecular class, and effects on patient prognosis. Gastroenterology 152(4): 745-761, 2017. PMID: 28043904. DOI: $10.1053 /$ j.gastro.2016.11.048

21 Liu CY, Chen KF and Chen PJ: Treatment of liver cancer. Cold Spring Harb Perspect Med 5(9): a021535, 2015. PMID: 26187874. DOI: $10.1101 /$ cshperspect.a021535

22 Zeng P, Li J, Chen Y and Zhang L: The structures and biological functions of polysaccharides from traditional chinese herbs. Prog Mol Biol Transl Sci 163: 423-444, 2019. PMID: 31030757. DOI: $10.1016 /$ bs.pmbts.2019.03.003

23 Singh A and Zhao K: Herb-drug interactions of commonly used chinese medicinal herbs. Int Rev Neurobiol 135: 197-232, 2017. PMID: 28807159. DOI: 10.1016/bs.irn.2017.02.010

24 Tay KC, Tan LT, Chan CK, Hong SL, Chan KG, Yap WH, Pusparajah P, Lee LH and Goh BH: Formononetin: A review of its anticancer potentials and mechanisms. Front Pharmacol 10: 820, 2019. PMID: 31402861. DOI: 10.3389/fphar.2019.00820

25 Rauf A, Imran M, Khan IA, Ur-Rehman M, Gilani SA, Mehmood $\mathrm{Z}$ and Mubarak MS: Anticancer potential of quercetin: A comprehensive review. Phytother Res 32(11): 21092130, 2018. PMID: 30039547. DOI: 10.1002/ptr.6155

$26 \mathrm{Ren} \mathrm{M}, \mathrm{Xu} \mathrm{W}$ and $\mathrm{Xu} \mathrm{T}$ : Salidroside represses proliferation, migration and invasion of human lung cancer cells through akt and mek/erk signal pathway. Artif Cells Nanomed Biotechnol 47(1): 1014-1021, 2019. PMID: 30880481. DOI: 10.1080/21691401.2019.1584566

27 Chen Z, Zhu R, Zheng J, Chen C, Huang C, Ma J, Xu C, Zhai $\mathrm{W}$ and Zheng J: Cryptotanshinone inhibits proliferation yet induces apoptosis by suppressing stat 3 signals in renal cell carcinoma. Oncotarget 8(30): 50023-50033, 2017. PMID: 28654902. DOI: $10.18632 /$ oncotarget.18483

28 Ting CY, Wang HE, Yu CC, Liu HC, Liu YC and Chiang IT: Curcumin triggers DNA damage and inhibits expression of DNA repair proteins in human lung cancer cells. Anticancer Res 35(7): 3867-3873, 2015. PMID: 26124332.

29 Yu T, Dohl J, Elenberg F, Chen Y and Deuster P: Curcumin induces concentration-dependent alterations in mitochondrial function through ros in c2c12 mouse myoblasts. J Cell Physiol 234(5): 63716381, 2019. PMID: 30246249 . DOI: 10.1002/jcp.27370

30 Xia S: Biological mechanisms and therapeutic relevance of the gasdermin family. Mol Aspects Med, 2020. PMID: 32800355. DOI: $10.1016 /$ j.mam.2020.100890
Received October 19, 2020

Revised November 2, 2020

Accepted November 3, 2020 